\title{
A fractional step method on a special mesh for the resolution of multidimensional evolutionary convection-diffusion problems
}

\author{
C. Clavero ${ }^{\mathrm{a}, *, 1,2}$, J.C. Jorge ${ }^{\mathrm{b}, 1,2}$, F. Lisbona ${ }^{\mathrm{a}, 1}$, G.I. Shishkin ${ }^{\mathrm{c}, 3}$ \\ ${ }^{a}$ Departamento de Matemática Aplicada, Universidad de Zaragoza, Zaragoza, Spain \\ ${ }^{\mathrm{b}}$ Departamento de Matemática e Informática, Universidad Pública de Navarra, Pamplona, Spain \\ ${ }^{\mathrm{c}}$ Institute of Mathematics and Mechanics, Ekaterinburg, Russia
}

\begin{abstract}
In this paper we consider numerical schemes for multidimensional evolutionary convection-diffusion problems, where the approximation properties are uniform in the diffusion parameter. In order to obtain an efficient method, to provide good approximations with independence of the size of the diffusion parameter, we have developed a numerical method which combines a finite difference spatial discretization on a special mesh and a fractional step method for the time variable. The special mesh allows a correct approximation of the solution in the boundary layers, while the fractional steps permits a low computational cost algorithm. Some numerical examples confirming the expected behavior of the method are shown. ๑ 1998 Elsevier Science B.V. and IMACS. All rights reserved.
\end{abstract}

Keywords: Singular perturbation; Alternating directions; Uniform convergence

\section{Introduction}

In this paper we are concerned with the numerical solution of convection-diffusion parabolic problems, governed by the equation

$$
\frac{\partial u}{\partial t}-\varepsilon \Delta u+\vec{v} \vec{\nabla} u+k u=f(x, y, t),
$$

where $\varepsilon$ is a small parameter $0<\varepsilon \leqslant 1$ and $\vec{v}=\left(v_{1}(x, y), v_{2}(x, y)\right), k=k_{1}(x, y)+k_{2}(x, y)$ and $f=f(x, y, t)$ are smooth functions, with $v_{i}>\bar{v}>0$, and $k_{i} \geqslant 0$ for $i=1,2$.

It is known that, generally, the solutions of these problems present a multiscale character even for smooth data, i.e., such solutions vary rapidly in certain narrow regions called layers (for an account of

\footnotetext{
* Corresponding author. E-mail: clavero@posta.unizar.es.

${ }^{1}$ Research partially supported by the CICYT project No. AMB94-0396.

${ }^{2}$ Research partially supported by a project of Gobierno de Navarra.

${ }^{3}$ Research partially supported by the Russian Foundation for Basic Research.
} 
asymptotic results about this kind of problems see $[14,16,18])$. This behavior causes very inaccurate numerical solutions if standard finite difference or finite element methods are used on uniform meshes, unless a large number ( $\varepsilon$-dependent) of mesh points is considered.

Such drawback appears even for very simple (one-dimensional and stationary) singular perturbation problems of this kind, and has led to the development of adaptive techniques, capable of producing good approximations on meshes with a number of grid points independent of $\varepsilon$. In this context, the uniform convergence is the key property; it means that the rate of convergence and the error constant of the method are independent of the singular perturbation parameter $\varepsilon$.

Two different routes to construct uniformly convergent schemes have been followed in recent years: firstly, the use of exponentially fitted schemes, which have coefficients of exponential type adapted to the singular perturbation problems (see $[5,6,17]$ ); secondly, the special mesh approach (see $[7,10,16])$, which constructs meshes adapted to the solution of the problem. In these two contexts, several schemes for one-dimensional stationary problems have been deeply studied. This kind of analysis is, however, a difficult task in the case of multidimensional stationary or evolutionary problems.

The difficulties of the development of exponential fitting schemes for multidimensional problems, encouraged us to consider, in some earlier papers, see $[1,2]$, an alternating direction method. In this way, we took advantage of known techniques for uniform convergence of one-dimensional exponentialfitting schemes, and also of the computational cost reduction yielded by the use of alternating directions. Some low-cost uniformly convergent methods were developed for certain multidimensional singularly perturbed parabolic problems.

Recently, a simple type of special non-uniform meshes (see [19-21]) has permitted the construction of uniformly convergent methods using standard stable discretizations. In general, it is not easy to prove the uniform convergence results that the numerical experiments show. In work of Sun and Stynes (see [22,23]) weak energy norms, finite element methods and special meshes for one-dimensional problems are used. In the paper [24] of Ross and Stynes, uniform convergence of an upwind type method is proved. For two-dimensional stationary problems, the papers of Hegarty et al. [8,9] and Clavero et al. [4], present some numerical results obtained using two-dimensional Shishkin meshes for regular and parabolic layers. The book of Miller et al. [16] gives the most recent results on numerical approximation of singularly perturbed problems on Shishkin meshes. The method that we propose in this paper uses some of these special meshes, which are appropriate for discretizing the type of one-dimensional problems resulting from the time discretization process.

The remainder of this section is devoted to a detailed description of the algorithm. In Section 2, under certain hypotheses on the smoothness and the asymptotic structure of the exact solution, we prove the uniform convergence of the method. In Section 3, we prove some results, which are needed in Section 2, concerning the $L^{\infty}$-uniform convergence of the simple upwind method on special meshes. In Section 4, some numerical results are given. These ones illustrate how well the proposed algorithm works. Finally, in Appendix A we give the outlines of some results concerning the behavior of exact solutions of continuous and semidiscrete problems and their derivatives.

For simplicity, we shall consider the following initial boundary value problem:

$$
\begin{array}{ll}
\frac{\partial u}{\partial t}-\varepsilon \Delta u+\vec{v} \vec{\nabla} u+k u=f & \text { in } \Omega \times[0, T] \equiv(0,1) \times(0,1) \times(0, T], \\
u(x, y, 0)=u_{0}(x, y) & \text { in } \Omega, \\
u(x, y, t)=0 & \text { in } \partial \Omega \times(0, T] .
\end{array}
$$


Throughout this paper we set

$$
\begin{aligned}
& L_{x, \varepsilon} \equiv-\varepsilon \frac{\partial^{2}}{\partial x^{2}}+v_{1} \frac{\partial}{\partial x}+k_{1}, \\
& L_{y, \varepsilon} \equiv-\varepsilon \frac{\partial^{2}}{\partial y^{2}}+v_{2} \frac{\partial}{\partial y}+k_{2} .
\end{aligned}
$$

The operators $L_{x, \varepsilon}$ can be considered as a family of one-dimensional differential operators with one parameter $y \in(0,1)$ (similar comments can be applied to $L_{y, \varepsilon}$ ). Let us also also consider decompositions for the source term $f(x, y, t)=f_{1}(x, y, t)+f_{2}(x, y, t)$. As a first stage towards defining the algorithm, we introduce a time discretization process by means of the following fractional step scheme (see Jorge and Lisbona [11], Yanenko [25]):

$$
\begin{aligned}
& u^{0}=u_{0}(x, y), \\
& \left(I+\Delta t L_{x, \varepsilon}\right) u^{n+1 / 2}=u^{n}+\Delta t f_{1}\left(t_{n+1}\right), \quad y \in(0,1), \\
& u^{n+1 / 2}(0, y)=u^{n+1 / 2}(1, y)=0, \\
& \left(I+\Delta t L_{y, \varepsilon}\right) u^{n+1}=u^{n+1 / 2}+\Delta t f_{2}\left(t_{n+1}\right), \quad f_{1}+f_{2}=f, x \in(0,1), \\
& u^{n+1}(x, 0)=u^{n+1}(x, 1)=0,
\end{aligned}
$$

in such way that we could obtain semidiscrete approximations $u^{n}(x, y)$ to the solution $u(x, y, t)$ of (1.1) at the time levels $t_{n}=n \Delta t$, by solving exactly the elliptic problems of steps (1.4b), (1.4c). The second stage consists of obtaining appropriate approximations for (1.4b), (1.4c). To do that, we define a not necessarily uniform rectangular mesh $\Omega_{\varepsilon, h}$ as the tensor product $I_{x, \varepsilon, h} \times I_{y, \varepsilon, h}$ of one-dimensional Shishkin meshes, which will be generated in the following form. Let $h=1 / N$ with $\frac{1}{4} N \in \mathbb{N}$. Then, we take

$$
\sigma=\min \left\{\frac{1}{4}, m \varepsilon \log N\right\},
$$

where $m$ is a constant which we choose satisfying $m \geqslant 1 / \bar{v}$, we divide the interval $[0,1]$ into two subintervals $[0,1-\sigma]$ and $[1-\sigma, 1]$ and we define

$$
I_{x, \varepsilon, h} \equiv\left\{x_{0}, x_{1}, \ldots, x_{3 N / 4}=1-\sigma, \ldots, x_{N}\right\},
$$

with

$$
x_{i}= \begin{cases}i \frac{4(1-\sigma)}{3 N}, & i=0, \ldots, \frac{3}{4} N, \\ 1-\sigma+\left(i-\frac{3 N}{4}\right) \frac{4 \sigma}{N}, & i=\frac{3}{4} N+1, \ldots, N,\end{cases}
$$

(and analogously we can proceed for $I_{y, \varepsilon, h}$ ).

Using the notation $[\cdot]_{h}$ for the restriction of a function defined in $[0,1] \times[0,1]$ to $\Omega_{\varepsilon, h}$, we will compute the totally discrete approximations $u_{h}^{n}$ to $\left[u\left(t_{n}\right)\right]_{h}$ by

$$
\begin{aligned}
& u_{h}^{0}=\left[u_{0}\right]_{h}, \\
& \left(I+\Delta t L_{x, \varepsilon, h}\right) u_{h}^{n+1 / 2}=u_{h}^{n}+\Delta t\left[f_{1}\left(x, y, t_{n+1}\right)\right]_{h}, \quad y \in I_{y, \varepsilon, h}, \\
& u_{h}^{n+1 / 2}(0, y)=u_{h}^{n+1 / 2}(1, y)=0,
\end{aligned}
$$




$$
\begin{aligned}
& \left(I+\Delta t L_{y, \varepsilon, h}\right) u_{h}^{n+1}=u_{h}^{n+1 / 2}+\Delta t\left[f_{2}\left(x, y, t_{n+1}\right)\right]_{h}, \quad x \in I_{x, \varepsilon, h}, \\
& u_{h}^{n+1}(x, 0)=u_{h}^{n+1}(x, 1)=0,
\end{aligned}
$$

where $L_{x, \varepsilon, h}$ (and analogously $\left.L_{y, \varepsilon, h}\right)$ is the discretization of the differential operator $L_{x, \varepsilon}\left(L_{y, \varepsilon}\right)$ using the simple upwind finite difference scheme.

Finally, we want emphasize that each time step of (1.7) requires only the resolution of a set of uncoupled tridiagonal systems. Consequently, a low computational cost is needed by resolving with this algorithm. Furthermore, an easy and efficient implementation for parallel computation is possible.

Henceforth, we denote by $C$ any positive constant that is independent of parameters $\varepsilon, \Delta t$ and $N$.

\section{Convergence analysis}

We shall study firstly the convergence of the semidiscretization in time process (1.4).

Since the operators $\left(I+\Delta t L_{i, \varepsilon}\right), i=x, y$, satisfy a maximum principle, it is not difficult to deduce

$$
\left\|\left(I+\Delta t L_{i, \varepsilon}\right)^{-1}\right\|_{\infty} \leqslant \frac{1}{1+\bar{k}_{j} \Delta t},
$$

with $i=x, j=1$ or $i=y, j=2$ and $\bar{k}_{j}=\min _{(x, y) \in \Omega} k_{j} \geqslant 0, j=1,2$. This ensures the stability of scheme (1.4) (for more details see [2]). In order to analyze the convergence, we introduce the local error $e_{n+1}$ defined by

$$
e_{n+1}=u\left(t_{n+1}\right)-\widehat{u}^{n+1},
$$

where $\widehat{u}^{n+1}$ is the approximation to $u\left(t_{n+1}\right)$ given after a time step by (1.4), taking $u\left(t_{n}\right)$ as the starting value $u^{n}$, i.e.,

$$
\begin{aligned}
& u^{n}=u\left(t_{n}\right), \\
& \left(I+\Delta t L_{x, \bar{\varepsilon}}\right) \widehat{u}^{n+1 / 2}=u^{n}+\Delta t f_{1}\left(t_{n+1}\right), \\
& u^{n+1 / 2}(0, y)=u^{n+1 / 2}(1, y)=0, \\
& \left(I+\Delta t L_{y, \bar{\varepsilon}}\right) \widehat{u}^{n+1}=\widehat{u}^{n+1 / 2}+\Delta t f_{2}\left(t_{n+1}\right), \\
& u^{n+1}(x, 0)=u^{n+1}(x, 1)=0 .
\end{aligned}
$$

The following consistency result is obtained:

Lemma 2.1. Let us assume that

$$
\left\{u, \frac{\partial u}{\partial t}, \frac{\partial^{2} u}{\partial t^{2}}\right\} \subset C^{0}(\bar{\Omega} \times[0, T])
$$

and that they are bounded independently of $\varepsilon$, i.e.,

$$
\left|\frac{\partial^{i}}{\partial t^{i}} u(x, y, t)\right| \leqslant C, \quad(x, y, t) \in \bar{\Omega} \times[0, T], i \leqslant 2 .
$$

Then, the local error satisfies

$$
\left\|e_{n+1}\right\|_{\infty} \leqslant C(\Delta t)^{2} .
$$


In Appendix A we show briefly that it is possible to obtain (2.4) $\varepsilon$-independently if data are $\varepsilon$-independently smooth and compatible.

Combining (2.5) with the stability result (2.1), it is not difficult to show the following convergence result.

Theorem 2.2. Under assumptions of Lemma 2.1, we have

$$
\sup _{n \leqslant T /(\Delta t)}\left\|u\left(t_{n}\right)-u^{n}\right\|_{\infty} \leqslant C \Delta t
$$

For more details of the proofs of Lemma 2.1 and Theorem 2.2, see [1,2].

We study now the approximation properties of the spatial discretization process. To do that, we shall compare $\widehat{u}^{n+1}$ and $\widehat{u}_{h}^{n+1}$, the solutions obtained after a complete time step from (1.4) taking $u^{n} \equiv u\left(t_{n}\right)$, and from (1.7) taking $u_{h}^{n}=\left[u\left(t_{n}\right)\right]_{h}$, respectively.

Since the discrete operators $\left(I+\Delta t L_{i, \varepsilon, h}\right), i=x, y$, satisfy a maximum principle, we have

$$
\left\|\left(I+\Delta t L_{i, \varepsilon, h}\right)^{-1}\right\|_{\infty} \leqslant 1, \quad i=x, y,
$$

and therefore the total discretization is uniformly stable. Thus, we can prove the following theorem.

Theorem 2.3. Let us assume that $\widehat{u}^{n+1 / 2}$ and $\widehat{u}^{n+1}$ have the asymptotic behavior given by (3.2)-(3.4). Then, if we take $N^{-q} \leqslant C \Delta t$ with $0<q<1$, we have

$$
\left\|\left[\widehat{u}^{n+1}\right]_{h}-\widehat{u}_{h}^{n+1}\right\|_{\infty} \leqslant C \Delta t N^{q-1} \log N .
$$

The proof of this theorem consists of combining, in standard way, the stability result (2.1) and the uniform convergence results of Section 3 (see also [3]).

Finally, in order to prove the uniform convergence of the totally discrete scheme we split the global error in the form

$$
\left\|\left[u\left(t_{n}\right)\right]_{h}-u_{h}^{n}\right\|_{\infty} \leqslant\left\|\left[u\left(t_{n}\right)\right]_{h}-\left[\widehat{u}^{n}\right]_{h}\right\|_{\infty}+\left\|\left[\widehat{u}^{n}\right]_{h}-\widehat{u}_{h}^{n}\right\|_{\infty}+\left\|\widehat{u}_{h}^{n}-u_{h}^{n}\right\|_{\infty} .
$$

Combining the results of Lemma 2.1, Theorem 2.2 and (2.1), we obtain the following result (see [3]).

Theorem 2.4. Let $u$ be the solution of (1.1) and $\left\{u_{h}^{n}\right\}_{n}$ the solution of (1.7). Under the hypotheses of Lemma 2.1 and Theorem 2.2, there exists a constant $C$ such that

$$
\left\|\left[u\left(t_{n}\right)\right]_{h}-u_{h}^{n}\right\|_{\infty} \leqslant C\left(\Delta t+N^{q-1} \log N\right)
$$

with $0<q<1$.

\section{Analysis of spatial discretization}

In this section we study the approximation properties of the space discretization of the time semidiscrete problems (2.2). We only show the convergence of the numerical solution of problem

$$
\begin{aligned}
& \left(I+\Delta t L_{x, \varepsilon, h}\right) \widehat{u}_{h}^{n+1 / 2}=\left[u\left(x, y, t_{n}\right)\right]_{h}+\Delta t\left[f_{1}\left(x, y, t_{n+1}\right)\right]_{h}, \quad y \in I_{y, \varepsilon, h}, \\
& \widehat{u}_{h}^{n+1 / 2}(0, y)=\widehat{u}_{h}^{n+1 / 2}(1, y)=0
\end{aligned}
$$


to the exact solution of (2.2b), and similarly we can proceed for (2.2c).

Since the singular perturbation problems (2.2b) are essentially one-dimensional, they can be written in the form

$$
\begin{aligned}
& \left(I+\Delta t L_{x, \varepsilon}\right) w \equiv-\varepsilon \Delta t w^{\prime \prime}(x)+v_{1}(x, y) \Delta t w^{\prime}(x)+\left(1+\Delta t k_{1}(x, y)\right) w(x) \\
& =u\left(x, y, t_{n}\right)+\Delta t f_{1}\left(x, y, t_{n+1}\right), \quad 0<x<1, \\
& w(0)=0, \quad w(1)=0,
\end{aligned}
$$

where $y$ is a parameter $(0<y<1), w(x)=\widehat{u}^{n+1 / 2}(x, y), v_{1}(x, y)>\bar{v}>0, k_{1}(x, y) \geqslant 0$ and $v_{1}, k_{1}$, $f_{1}$ are sufficiently smooth in $\bar{\Omega}$. In the remainder of this section we will suppose (see Appendix A for justification) that $w \equiv w(x, \varepsilon)$ satisfies

$$
w(x, \varepsilon)=w_{1}(x, \varepsilon)+z(x, \varepsilon),
$$

where

$$
w_{1}(x, \varepsilon)=c_{y} \mathrm{e}^{-v_{1}(1, y)(1-x) / \varepsilon}, \quad\left|c_{y}\right| \leqslant C,
$$

and

$$
\left|z^{(i)}(x, \varepsilon)\right| \leqslant C\left(1+\varepsilon^{-i+1} \mathrm{e}^{-\bar{v}(1-x) / \varepsilon}\right), \quad 0 \leqslant i \leqslant 4 .
$$

To solve numerically (3.1) we consider the upwind difference scheme on the mesh $I_{x, \varepsilon, h}$ defined by (1.5), (1.6), which is piecewise uniform with $\frac{3}{4} N+1$ points in $[0,1-\sigma]$ and $\frac{1}{4} N+1$ points in $[1-\sigma, 1]$, and which is uniform if $\sigma=\frac{1}{4}$. Let us denote

$$
\begin{aligned}
& h_{j}=x_{j}-x_{j-1}, \quad j=1, \ldots, N, \quad \tilde{h}_{j}=\frac{h_{j}+h_{j+1}}{2}, \quad j=1, \ldots, N-1, \\
& H=\max _{j} h_{j} \quad \text { and } \quad \rho_{j}=\frac{h_{j}}{\varepsilon}, \quad j=1, \ldots, N .
\end{aligned}
$$

It holds

$$
h_{j}= \begin{cases}\frac{4(1-\sigma)}{3 N}, & j=1, \ldots, \frac{3}{4} N, \\ \frac{4 \sigma}{N}, & j=\frac{3}{4} N+1, \ldots, N .\end{cases}
$$

On this mesh the scheme is defined by

$$
\begin{aligned}
& \left(I+\Delta t L_{x, \varepsilon, h}\right) W_{j} \equiv r_{j}^{-} W_{j-1}+r_{j}^{c} W_{j}+r_{j}^{+} W_{j+1}=f_{j}, \quad j=1, \ldots, N-1, \\
& W_{0}=0, \quad W_{N}=0,
\end{aligned}
$$

with

$$
\begin{aligned}
& r_{j}^{-}=\frac{-\varepsilon \Delta t}{h_{j} \widetilde{h}_{j}}-\frac{v_{1, j} \Delta t}{h_{j}}, \quad r_{j}^{+}=\frac{-\varepsilon \Delta t}{h_{j+1} \widetilde{h}_{j}}, \quad r_{j}^{c}=1+\Delta t k_{1, j}-r_{j}^{-}-r_{j}^{+}, \\
& v_{1, j}=v_{1}\left(x_{j}, y\right), \quad k_{1, j}=k_{1}\left(x_{j}, y\right), \quad f_{j}=u\left(x_{j}, y, t_{n}\right)+\Delta t f_{1}\left(x_{j}, y, t_{n+1}\right) .
\end{aligned}
$$

In order to prove the uniform convergence of the method, we begin by studying its consistency. The local truncation error at an internal point of the mesh is introduced in standard way by

$$
\tau_{j}=\left(I+\Delta t L_{x, \varepsilon, h}\right)\left(w\left(x_{j}\right)\right)-\left(\left(I+\Delta t L_{x, \varepsilon}\right) w\right)\left(x_{j}\right), \quad j=1, \ldots, N-1 .
$$


We shall make use of Taylor expansions of functions $g(x)$ in a point $a$, with the following well-known expressions for the remainder:

$$
R_{n}(a, x, g)=g^{(n+1)}(\varphi) \frac{(x-a)^{n+1}}{(n+1) !}, \quad a<\varphi<x,
$$

and

$$
R_{n}(a, x, g)=\frac{1}{n !} \int_{a}^{x}(x-s)^{n} g^{(n+1)}(s) \mathrm{d} s .
$$

We shall also suppose that $\sigma=m \varepsilon \log N$ (in case $\sigma=\frac{1}{4}, h$ is small with respect to $\varepsilon$ and $\tau_{j}$ can be bounded in a classical way). Under this assumption, the mesh is not uniform and we study separately three cases depending on the localization of the point $x_{j}$ in the mesh.

Case 1. $0<x_{j}<1-\sigma$. Using Taylor expansions, it is straightforward that the local error is given by

$$
\tau_{j}=-\frac{h_{j}}{2} \Delta t v_{1, j} w^{\prime \prime}\left(x_{j}\right)+r_{j}^{-} R_{2}\left(x_{j}, x_{j-1}, w\right)+r_{j}^{+} R_{2}\left(x_{j}, x_{j+1}, w\right) .
$$

We distinguish two possibilities to find appropriate estimates of this error, depending on the value of $\rho_{j}$.

(i) If $\rho_{j} \leqslant 1$, using (3.2)-(3.4) to estimate the derivatives of $w$, the expression (3.9) for the remainder and the values of the coefficients $r_{j}^{-}, r_{j}^{+}$, it is easy to obtain

$$
\left|\tau_{j}\right| \leqslant C \Delta t h_{j}\left(1+\varepsilon^{-2} \mathrm{e}^{-\bar{v}\left(1-x_{j}\right) / \varepsilon}\right) .
$$

(ii) If $\rho_{j} \geqslant 1$, we use the decomposition (3.2) denoting by $\tau_{j}^{1}$ and $\tau_{j}^{2}$ the local errors corresponding to the functions $z(x, \varepsilon)$ and $w_{1}(x, \varepsilon)$, respectively. Using the estimates (3.4) and the integral form of the remainder (3.10), we deduce

$$
\begin{aligned}
\left|\frac{h_{j} \Delta t v_{1, j}}{2} z^{\prime \prime}\left(x_{j}\right)\right| \leqslant & C \Delta t h_{j}\left(1+\varepsilon^{-1} \mathrm{e}^{-\bar{v}\left(1-x_{j}\right) / \varepsilon}\right) \\
\left|r_{j}^{-} R_{2}\left(x_{j}, x_{j-1}, z\right)\right| & \leqslant C \Delta t \frac{\varepsilon+v_{1, j} h_{j}}{h_{j}^{2}} \int_{x_{j-1}}^{x_{j}}\left(x_{j-1}-s\right)^{2}\left(1+\varepsilon^{-2} \mathrm{e}^{-\bar{v}(1-s) / \varepsilon}\right) \mathrm{d} s \\
& \leqslant C \Delta t h_{j}\left(h_{j}+\varepsilon^{-1} \mathrm{e}^{-\bar{v}\left(1-x_{j}\right) / \varepsilon}\right) .
\end{aligned}
$$

Likewise,

$$
\left|r_{j}^{+} R_{2}\left(x_{j}, x_{j+1}, z\right)\right| \leqslant C \Delta t h_{j}+C \Delta t \mathrm{e}^{-\bar{v}\left(1-x_{j+1}\right) / \varepsilon} .
$$

Hence, using the three preceding estimates, we obtain

$$
\left|\tau_{j}^{1}\right| \leqslant C \Delta t h_{j}\left(1+\varepsilon^{-1} \mathrm{e}^{-\bar{v}\left(1-x_{j}\right) / \varepsilon}\right)+C \Delta t \mathrm{e}^{-\bar{v}\left(1-x_{j+1}\right) / \varepsilon} .
$$

To bound $\left|\tau_{j}^{2}\right|$, we proceed in a different way, since the function $w_{1}(x, \varepsilon)$ is known and we can calculate $\tau_{j}^{2}$ exactly. Then, 


$$
\begin{gathered}
\tau_{j}^{2}=w_{1}\left(x_{j}, \varepsilon\right)\left[r_{j}^{-}\left(\mathrm{e}^{-v_{1}(1, y) h_{j} / \varepsilon}-1\right)+r_{j}^{+}\left(\mathrm{e}^{v_{1}(1, y) h_{j+1} / \varepsilon}-1\right)\right. \\
\left.+\frac{\Delta t v_{1}(1, y)\left(v_{1}(1, y)-v_{1, j}\right)}{\varepsilon}\right]
\end{gathered}
$$

and using (3.7), it is easy to prove that

$$
\left|\tau_{j}^{2}\right| \leqslant \frac{C \Delta t}{h_{j}} \mathrm{e}^{-\bar{v}\left(1-x_{j+1}\right) / \varepsilon}+C \Delta t \varepsilon^{-1} \mathrm{e}^{-\bar{v}\left(1-x_{j}\right) / \varepsilon} .
$$

Finally,

$$
\begin{aligned}
\left|\tau_{j}\right| & \leqslant\left|\tau_{j}^{1}\right|+\left|\tau_{j}^{2}\right| \leqslant C \Delta t h_{j}+C \Delta t \varepsilon^{-1} \mathrm{e}^{-\bar{v}\left(1-x_{j}\right) / \varepsilon}+\frac{C \Delta t}{h_{j}} \mathrm{e}^{-\bar{v}\left(1-x_{j+1}\right) / \varepsilon} \\
& =C \Delta t h_{j}+\frac{C \Delta t}{h_{j+1}} h_{j+1} \varepsilon^{-1} \mathrm{e}^{-\bar{v} h_{j+1} / \varepsilon} \mathrm{e}^{-\bar{v}\left(1-x_{j+1}\right) / \varepsilon}+\frac{C \Delta t}{h_{j}} \mathrm{e}^{-\bar{v}\left(1-x_{j+1}\right) / \varepsilon} \\
& \leqslant C \Delta t h_{j}+\frac{C \Delta t}{h_{j}} \mathrm{e}^{-\bar{v}\left(1-x_{j+1}\right) / \varepsilon}
\end{aligned}
$$

since $h_{j}=h_{j+1}$ and

$$
\frac{h_{j+1}}{\varepsilon} \mathrm{e}^{-\bar{v} h_{j+1} / \varepsilon} \leqslant C \text {. }
$$

Case 2. $1-\sigma<x_{j}<1$. In this case the study of $\tau_{j}$ is straightforward. Using the expression (3.11) for the local error, the estimates for the exact solution given in (3.2)-(3.4) and (3.9) for the remainder of the Taylor expansions, we obtain

$$
\left|\tau_{j}\right| \leqslant C \Delta t h_{j}\left(1+\varepsilon^{-2} \mathrm{e}^{-\bar{v}\left(1-x_{j}\right) / \varepsilon}\right) .
$$

Case 3. $x_{j}=1-\sigma$. Here, we shall distinguish again two possibilities depending on the value of $\rho_{j}$.

(i) If $\rho_{j} \leqslant 1$, similar arguments to those used in Case 1(i) permit us to prove

$$
\left|\tau_{j}\right| \leqslant C \Delta t h_{j}\left(1+\varepsilon^{-2} \mathrm{e}^{-\bar{v}\left(1-x_{j}\right) / \varepsilon}\right) .
$$

(ii) If $\rho_{j} \geqslant 1$, we write the local error in the form

$$
\tau_{j}=r_{j}^{-} R_{1}\left(x_{j}, x_{j-1}, w\right)+r_{j}^{+} R_{1}\left(x_{j}, x_{j+1}, w\right)+\Delta t \varepsilon w^{\prime \prime}\left(x_{j}\right)
$$

and we denote by $\tau_{j}^{1}, \tau_{j}^{2}$ the same parts of error as in Case 1(ii).

Using the estimates given by (3.4), the remainder of the Taylor expansion in the form (3.10) and the expressions (3.7) for the coefficients, we have

$$
\begin{gathered}
\left|\Delta t \varepsilon z^{\prime \prime}\left(x_{j}\right)\right| \leqslant C \Delta t \varepsilon\left(1+\varepsilon^{-1} \mathrm{e}^{-\bar{v}\left(1-x_{j}\right) / \varepsilon}\right) \leqslant C \Delta t h_{j}+C \Delta t \mathrm{e}^{-\bar{v}\left(1-x_{j}\right) / \varepsilon} \\
\left|r_{j}^{-} R_{1}\left(x_{j}, x_{j-1}, z\right)\right| \leqslant C \Delta t \frac{\varepsilon+v_{1, j} h_{j}}{h_{j}^{2}} \int_{x_{j-1}}^{x_{j}}\left|\left(x_{j-1}-s\right)\right|\left(1+\varepsilon^{-1} \mathrm{e}^{-\bar{v}(1-s) / \varepsilon}\right) \mathrm{d} s \\
\leqslant C \Delta t h_{j}+C \Delta t \mathrm{e}^{-\bar{v}\left(1-x_{j}\right) / \varepsilon},
\end{gathered}
$$


and

$$
\begin{aligned}
\left|r_{j}^{+} R_{1}\left(x_{j}, x_{j+1}, z\right)\right| & \leqslant C \Delta t \frac{\varepsilon}{h_{j}^{2}} \int_{x_{j}}^{x_{j+1}}\left|\left(x_{j+1}-s\right)\right|\left(1+\varepsilon^{-1} \mathrm{e}^{-\bar{v}(1-s) / \varepsilon}\right) \mathrm{d} s \\
& \leqslant C \Delta t h_{j}+C \Delta t \mathrm{e}^{-\bar{v}\left(1-x_{j+1}\right) / \varepsilon} .
\end{aligned}
$$

From the three preceding estimates, we obtain

$$
\left|\tau_{j}^{1}\right| \leqslant C \Delta t h_{j}+C \Delta t \mathrm{e}^{-\bar{v}\left(1-x_{j+1}\right) / \varepsilon} .
$$

To bound $\tau_{j}^{2}$, we use (3.13) obtaining

$$
\left|r_{j}^{-}\left(\mathrm{e}^{-v_{1}(1, y) h_{j} / \varepsilon}-1\right)+r_{j}^{+}\left(\mathrm{e}^{v_{1}(1, y) h_{j+1} / \varepsilon}-1\right)\right| \leqslant \frac{C \Delta t}{h_{j}},
$$

and

$$
\left|\Delta t \frac{v_{1}(1, y)\left(v_{1}(1, y)-v_{1, j}\right)}{\varepsilon}\right| \leqslant \frac{C \Delta t \sigma}{\varepsilon} \leqslant \frac{C \Delta t}{h_{j}} .
$$

In the last inequality we have taken into account that $h_{j}=4(1-\sigma) /(3 N), \sigma=m \varepsilon \log N$ and $4(1-\sigma) \log N \leqslant C N$. Therefore,

$$
\left|\tau_{j}^{2}\right| \leqslant \frac{C \Delta t}{h_{j}} \mathrm{e}^{-\bar{v}\left(1-x_{j}\right) / \varepsilon} .
$$

Finally, from the last estimates for $\left|\tau_{j}^{1}\right|$ and $\left|\tau_{j}^{2}\right|$, we deduce that

$$
\left|\tau_{j}\right| \leqslant C \Delta t h_{j}+\frac{C \Delta t}{h_{j}} \mathrm{e}^{-\bar{v}\left(1-x_{j}\right) / \varepsilon}+C \Delta t \mathrm{e}^{-\bar{v}\left(1-x_{j+1}\right) / \varepsilon} \leqslant C \Delta t h_{j}+\frac{C \Delta t}{h_{j}} \mathrm{e}^{-\bar{v}\left(1-x_{j+1}\right) / \varepsilon} .
$$

Let us remark that we have just found estimates for the local error that are not uniform in $\varepsilon$. To obtain an uniform convergence result we shall instead use the barrier function technique (see Kellogg and Tsan [12]). Thus, let us define

$$
\Phi_{1, j}=1+x_{j}, \quad \Phi_{2, j}(\beta)=\frac{1}{\prod_{i=j+1}^{N} \mu_{i}(\beta)}, \quad \mu_{i}(\beta)=1+\frac{\beta h_{i}}{\varepsilon},
$$

with $\beta$ a constant to be fixed later. Applying the finite difference operator to the barrier functions, we have the following estimates:

$$
\begin{aligned}
\left(I+\Delta t L_{x, \varepsilon, h}\right)\left(\Phi_{1, j}(\beta)\right) & =\left(1+\Delta t k_{1, j}\right)\left(1+x_{j}\right)+v_{1, j} \Delta t>\Delta t \bar{v}>0, \\
\left(I+\Delta t L_{x, \varepsilon, h}\right)\left(\Phi_{2, j}(\beta)\right) & =r_{j}^{-} \Phi_{2, j-1}(\beta)+r_{j}^{c} \Phi_{2, j}(\beta)+r_{j}^{+} \Phi_{2, j+1}(\beta) \\
& \geqslant \Phi_{2, j}(\beta)\left[r_{j}^{-}\left(\frac{1}{\mu_{j}(\beta)}-1\right)+r_{j}^{+}\left(u_{j+1}(\beta)-1\right)\right] .
\end{aligned}
$$

Simple calculations render

$$
\left(I+\Delta t L_{x, \varepsilon, h}\right)\left(\Phi_{2, j}(\beta)\right) \geqslant \Phi_{2, j}(\beta) \frac{\beta \Delta t}{\widetilde{h}_{j}} \frac{v_{1, j} \widetilde{h}_{j}-\beta h_{j}}{\varepsilon+\beta h_{j}} .
$$

For a deeper study of these estimates, we shall distinguish the same cases as in the previous study of the local error. 
Case 1. Note that $h_{j}=h_{j+1}=\widetilde{h}_{j}$. Using (3.19), we obtain

$$
\left(I+\Delta t L_{x, \varepsilon, h}\right)\left(\Phi_{2, j}(\beta)\right) \geqslant \frac{C(\beta) \Delta t}{\max \left\{\varepsilon, h_{j}\right\}} \Phi_{2, j}(\beta),
$$

with $\beta \leqslant \bar{v}$.

Case 2. Again, $h_{j}=h_{j+1}=\widetilde{h}_{j}$ and using (3.19) we deduce (3.20), if condition $\beta \leqslant \bar{v}$ is satisfied.

Case 3. Now using (3.19) we have

$$
\left(I+\Delta t L_{x, \varepsilon, h}\right)\left(\Phi_{2, j}(\beta)\right) \geqslant \Phi_{2, j}(\beta) \frac{\beta \Delta t}{\widetilde{h}_{j}} \frac{\left(v_{1, j}-2 \beta\right) h_{j}}{2\left(\varepsilon+\beta h_{j}\right)} \geqslant \frac{C(\beta) \Delta t}{\max \left\{\varepsilon, h_{j}\right\}} \Phi_{2, j}(\beta),
$$

where the last inequality holds only if $\beta \leqslant \bar{v} / 2$. Hence, under condition $\beta \leqslant \bar{v} / 2$, in all cases we have proved that

$$
\left(I+\Delta t L_{x, \varepsilon, h}\right)\left(\Phi_{2, j}(\beta)\right) \geqslant \frac{C \Delta t}{\max \left\{\varepsilon, h_{j}\right\}} \Phi_{2, j}(\beta) .
$$

In order to combine the estimates obtained for the local truncation error with the estimates for the barrier functions, to prove the uniform convergence of the simple upwind scheme, we will use the following technical result.

Lemma 3.1. For all $\beta>0$ we have:

(i) $\Phi_{2, j}(\beta) \geqslant \mathrm{e}^{-\beta\left(1-x_{j}\right) / \varepsilon}$.

(ii) If $h_{i} \leqslant \varepsilon$ for $i \geqslant j+1$ then

$$
\frac{1}{\prod_{i=j+1}^{N} \mu_{i}(\beta)} \leqslant \frac{C}{\prod_{i=j+1}^{N} \mathrm{e}^{2 \beta h_{i} / \varepsilon}} .
$$

Theorem 3.2. Let $w(x, \varepsilon)$ be the exact solution of problem (3.1) and $\left\{W_{j}\right\}$ the numerical solution of upwind scheme (3.5)-(3.8) defined on the special mesh $I_{x, \varepsilon, h}$ given by (1.5), (1.6). Then, if we choose $m \geqslant 1 / \bar{v}$ and $\beta=\bar{v} / 2$, there exists a positive constant $C$ independent of $y, \varepsilon$ and $N$, such that

$$
\left|w\left(x_{j}, \varepsilon\right)-W_{j}\right| \leqslant C N^{-1} \log N
$$

Proof. Let us denote by $e_{j}=w\left(x_{j}, \varepsilon\right)-W_{j}$ the global error in $x_{j}$. To bound $\left|e_{j}\right|$, we shall distinguish two cases. If $\rho_{j} \leqslant 1$, we take

$$
K_{1}\left(h_{j}, \varepsilon\right)=C h_{j}, \quad K_{2}\left(h_{j}, \varepsilon\right)=C \frac{h_{j}}{\varepsilon} .
$$

Then, using the estimates (3.12) and (3.15)-(3.17) for the local error, the estimates given in (3.21) and Lemma 3.1, we deduce

$$
\left(I+\Delta t L_{x, \varepsilon, h}\right)\left(K_{1} \Phi_{1, j}(\beta)+K_{2} \Phi_{2, j}(\beta)\right) \geqslant\left|\tau_{j}\right|
$$


and using the discrete maximum principle for the operator $\left(I+\Delta t L_{x, \varepsilon, h}\right)$ and the condition $\beta m \geqslant 1$, we obtain

$$
\left|e_{j}\right| \leqslant C h_{j}+C \frac{h_{j}}{\varepsilon} \Phi_{2, j}(\beta) \leqslant C N^{-1} \log N
$$

If $\rho_{j} \geqslant 1$, taking

$$
K_{1}\left(h_{j}, \varepsilon\right)=C h_{j}, \quad K_{2}\left(h_{j}, \varepsilon\right)=C \mu_{j+1}(\beta),
$$

we deduce again

$$
\left(I+\Delta t L_{x, \varepsilon, h}\right)\left(K_{1} \Phi_{1, j}(\beta)+K_{2} \Phi_{2, j}(\beta)\right) \geqslant\left|\tau_{j}\right|,
$$

so, the maximum principle permit us to prove

$$
\left|e_{j}\right| \leqslant C h_{j}+\frac{C}{N^{2 \beta m}} \leqslant C N^{-1}
$$

Note that under the same hypotheses of Theorem 2.4, if we impose $N^{-q} \leqslant C \Delta t$, with $0<q<1$, we obtain

$$
\left|w\left(x_{j}, \varepsilon\right)-W_{j}\right| \leqslant C \Delta t N^{-1+q} \log N,
$$

which is the required result in Section 2.

\section{Numerical results}

In this section we show some numerical results obtained in the integration of two problems of type (1.1). In both cases we use a constant time step and special meshes in the spatial variables. We have first considered an example with known exact solution in order to compute exactly the pointwise errors

$$
\mathrm{e}_{\varepsilon}^{N, \Delta t}(i, j, n)=\left|u_{\varepsilon}\left(x_{i}, y_{j}, t_{n}\right)-u^{N}\left(x_{i}, y_{j}, t_{n}\right)\right|,
$$

where the superscript $N$ indicates the number of mesh points used in the $x$ - and $y$-directions, and $\Delta t$ the stepsize in time. For each $\varepsilon$ the maximum nodal error is given by

$$
E_{\varepsilon, N, \Delta t}=\max _{i, j, n} \mathrm{e}_{\varepsilon}^{N, \Delta t}(i, j, n)
$$

and, for each $N$ and $\Delta t$, the $\varepsilon$-uniform maximum nodal error is defined by

$$
E_{N, \Delta t}=\max _{\varepsilon} E_{\varepsilon, N, \Delta t} \text {. }
$$

Computed values of $E_{\varepsilon, N, \Delta t}$ and $E_{N, \Delta t}$ for this problem are given in Table 1 for various values of $\varepsilon$, $N$ and $\Delta t$.

A second example whose exact solution is not known is also considered. In this case, the pointwise error $\mathrm{e}_{\varepsilon}^{N, \Delta t}(i, j, n)$ is estimated by $\mathrm{e}_{\varepsilon}^{N, \Delta t, *}(i, j, n)=\left|\bar{u}\left(x_{i}, y_{j}, t_{n}\right)-u^{N}\left(x_{i}, y_{j}, t_{n}\right)\right|$ where $\bar{u}\left(x_{i}, y_{j}, t_{n}\right)$ denote the numerical solution obtained by the method using a special mesh with $N=256$ points in each spatial direction and $\Delta t=0.003125$. Then, in a similar way as before, we define $E_{\varepsilon, N, \Delta t}^{*}$ and $E_{N, \Delta t}^{*}$. Note that the meshes are not uniform, thus we use a bilinear interpolation to obtain $\bar{u}$ on the coarse mesh. 
To obtain a numerical $\varepsilon$-uniform rate of convergence, we proceed in a similar way to the doublemesh principle (see, e.g., Hegarty et al. [8]), but doubling the number of points in the mesh instead of half the mesh size. Then, the numerical order of convergence is given by

$$
p=\frac{\log \left(E_{\varepsilon, N, \Delta t} / E_{\varepsilon, 2 N, \Delta t}\right)}{\log 2} \quad \text { or } \quad p=\frac{\log \left(E_{\varepsilon, N, \Delta t}^{*} / E_{\varepsilon, 2 N, \Delta t}^{*}\right)}{\log 2},
$$

if the exact solution is known or unknown, respectively. We recall that a numerical method for solving (1.1) is said to have an $\varepsilon$-uniform rate of convergence of order $p$ on the sequence of meshes $\left\{\Omega_{N}\right\}_{1}^{\infty} \times\left\{t_{n}\right\}_{0}^{T}$ if there exist $N_{0}$ and $\Delta t_{0}$, independent of $\varepsilon$, such that for all $N \geqslant N_{0}$ and $\Delta t \leqslant \Delta t_{0}$

$$
\sup _{0 \leqslant t_{n} \leqslant T}\left(\sup _{0<\varepsilon \leqslant 1}\left\|u-u^{N}\right\|_{\Omega_{N}, t_{n}}\right) \leqslant C\left(\Delta t+N^{-1}\right)^{p}
$$

$\left(\|w\|_{\Omega_{N}, t_{n}} \equiv \max _{\left(x_{i}, y_{j}\right) \in \Omega_{N}}\left|w\left(x_{i}, y_{j}, t_{n}\right)\right|\right)$, where $u$ is the solution of (1.1), $u^{N}$ is the numerical approximation to $u$ and $C$ and $p>0$ are independent of $\varepsilon, \Delta t$ and $N$.

In the numerical examples below, we choose the following decomposition of the function $f$ :

$$
\begin{aligned}
& f_{1}(x, y, t)=f(x, y, t)-f_{2}(x, y, t), \\
& f_{2}(x, y, t)=f(x, 0, t)+y(f(x, 1, t)-f(x, 0, t)) .
\end{aligned}
$$

With this special decomposition, the hypothesis

$$
f(0,0, t)=f(0,1, t)=f(1,0, t)=f(1,1, t)=0
$$

clearly gives

$$
f_{1}(x, 0, t)=f_{1}(x, 1, t)=0, \quad f_{2}(0, y, t)=f_{2}(1, y, t)=0 .
$$

This property is needed for us to prove the asymptotic behavior of exact solution of problem (1.1) and semidiscrete problems (3.1) (see Appendix A).

The mesh refinement strategy that we have used for our numerical experiments, fails to satisfy the hypothesis $N^{-q} \leqslant C \Delta t$ at the limit. However, the obtained results show a reduction of the error which gives the convergence of the method. Therefore, this hypothesis seems to be unnecessary for convergence or order given in Theorem 2.4. On the other hand, the influence of $q$ in the estimated order of convergence would not exist if the hypothesis reveals unnecessary. Anyway, the presence of $\log N$ makes it difficult to appreciate if there is an actual reduction from $N^{-1} \log N$ to $N^{-1+q} \log N$. This difficulty would appear even if we take the care of making all meshes satisfying the restriction above.

We want also remark that, from both theoretical and numerical points of view, the choice of decomposition $k=k_{1}+k_{2}$ ( $k_{i}$ smooth and positive) only affects to the error constant $C$ of the global error.

The numerical simulations have been performed on a DEC 3000 Model 500/S with OpenVMS.

\section{Example 1.}

$$
\begin{array}{ll}
u_{t}-\varepsilon \Delta u+(1+x) u_{x}+(2-y) u_{y}+\left(x^{2}+y^{2}+1\right) u=f, & (x, y, t) \in \Omega \times(0,2], \\
u(x, y, t)=0 & \text { in } \partial \Omega \times(0,2], \\
u(x, y, 0)=0, & (x, y) \in \Omega,
\end{array}
$$


Table 1

Maximum nodal errors $E_{\varepsilon, N, \Delta t}$ and $E_{N, \Delta t}$ for Example 1

\begin{tabular}{|c|c|c|c|c|c|c|}
\hline$\varepsilon$ & $\begin{array}{c}N=8 \\
\Delta t=0.1\end{array}$ & $\begin{array}{c}N=16 \\
\Delta t=0.05\end{array}$ & $\begin{array}{c}N=32 \\
\Delta t=0.025\end{array}$ & $\begin{array}{c}N=64 \\
\Delta t=0.0125\end{array}$ & $\begin{array}{c}N=128 \\
\Delta t=0.00625\end{array}$ & $\begin{array}{c}N=256 \\
\Delta t=0.003125\end{array}$ \\
\hline 1 & $7.921 \mathrm{E}-3$ & $4.803 \mathrm{E}-3$ & $2.678 \mathrm{E}-3$ & $1.418 \mathrm{E}-3$ & $7.306 \mathrm{E}-4$ & $3.709 \mathrm{E}-4$ \\
\hline $10^{-1}$ & $1.252 \mathrm{E}-1$ & $9.826 \mathrm{E}-2$ & $6.117 \mathrm{E}-2$ & $3.434 \mathrm{E}-2$ & $1.835 \mathrm{E}-2$ & $9.501 \mathrm{E}-3$ \\
\hline $10^{-2}$ & $2.317 \mathrm{E}-1$ & $1.794 \mathrm{E}-1$ & $1.251 \mathrm{E}-1$ & $8.124 \mathrm{E}-2$ & $5.072 \mathrm{E}-2$ & $3.014 \mathrm{E}-2$ \\
\hline $10^{-3}$ & $2.506 \mathrm{E}-1$ & $1.986 \mathrm{E}-1$ & $1.430 \mathrm{E}-1$ & $9.340 \mathrm{E}-2$ & $5.760 \mathrm{E}-2$ & $3.395 \mathrm{E}-2$ \\
\hline $10^{-4}$ & $2.526 \mathrm{E}-1$ & $2.008 \mathrm{E}-1$ & $1.455 \mathrm{E}-1$ & $9.549 \mathrm{E}-2$ & $5.915 \mathrm{E}-2$ & $3.508 \mathrm{E}-2$ \\
\hline $10^{-5}$ & $2.528 \mathrm{E}-1$ & $2.011 \mathrm{E}-1$ & $1.457 \mathrm{E}-1$ & $9.574 \mathrm{E}-2$ & $5.933 \mathrm{E}-2$ & $3.523 \mathrm{E}-2$ \\
\hline $10^{-6}$ & $2.528 \mathrm{E}-1$ & $2.011 \mathrm{E}-1$ & $1.457 \mathrm{E}-1$ & $9.574 \mathrm{E}-2$ & $5.935 \mathrm{E}-2$ & $3.524 \mathrm{E}-2$ \\
\hline $10^{-7}$ & $2.528 \mathrm{E}-1$ & $2.011 \mathrm{E}-1$ & $1.457 \mathrm{E}-1$ & $9.574 \mathrm{E}-2$ & $5.935 \mathrm{E}-2$ & $3.524 \mathrm{E}-2$ \\
\hline$E_{N, \Delta t}$ & 0.2528 & 0.2011 & 0.1457 & 0.0957 & 0.0593 & 0.0352 \\
\hline
\end{tabular}

Table 2

Numerical order of convergence for Example 1

\begin{tabular}{cccccc}
\hline$\varepsilon$ & $N=8$ & $N=16$ & $N=32$ & $N=64$ & 0.956 \\
\hline 1 & 0.721 & 0.842 & 0.917 & 0.904 & 0.949 \\
$10^{-1}$ & 0.349 & 0.683 & 0.832 & 0.679 & 0.750 \\
$10^{-2}$ & 0.369 & 0.520 & 0.622 & 0.697 & 0.762 \\
$10^{-3}$ & 0.335 & 0.473 & 0.614 & 0.690 & 0.753 \\
$10^{-4}$ & 0.331 & 0.464 & 0.607 & 0.690 & 0.752 \\
$10^{-5}$ & 0.330 & 0.464 & 0.605 & 0.689 & 0.752 \\
$10^{-6}$ & 0.330 & 0.464 & 0.607 & 0.689 & 0.752 \\
$10^{-7}$ & 0.330 & 0.464 & 0.607 & 0.679 & 0.750 \\
Min. & 0.330 & 0.464 & 0.65 & &
\end{tabular}

where $f$ is such that the exact solution is given by

$$
u_{\varepsilon}(x, y, t)=\mathrm{e}^{-t} x y\left(h_{1}(x)-1\right)\left(h_{2}(y)-1\right),
$$

with

$$
h_{1}(\zeta)=\mathrm{e}^{\left(-3-2 \zeta-\zeta^{2}\right) /(2 \varepsilon)}, \quad h_{2}(\zeta)=\mathrm{e}^{-\left(3-4 \zeta+\zeta^{2}\right) /(2 \varepsilon)} .
$$

\section{Example 2.}

$$
\begin{array}{ll}
u_{t}-\varepsilon \Delta u+\left(1-\frac{x y}{2}\right) u_{x}+\left(1+\frac{x y}{2}\right) u_{y}=f(x, y), & (x, y, t) \in \Omega \times(0,2], \\
u(x, y, t)=0 & \text { in } \partial \Omega \times(0,2], \\
u(x, y, 0)=0, & (x, y) \in \Omega,
\end{array}
$$


Table 3

Maximum nodal errors $E_{\varepsilon, N, \Delta t}^{*}$ and $E_{N, \Delta t}^{*}$ for Example 2

\begin{tabular}{|c|c|c|c|c|c|}
\hline$\varepsilon$ & $\begin{array}{c}N=8 \\
\Delta t=0.1\end{array}$ & $\begin{array}{c}N=16 \\
\Delta t=0.05\end{array}$ & $\begin{array}{c}N=32 \\
\Delta t=0.025\end{array}$ & $\begin{array}{c}N=64 \\
\Delta t=0.0125\end{array}$ & $\begin{array}{c}N=128 \\
\Delta t=0.00625\end{array}$ \\
\hline 1 & $1.010 \mathrm{E}-2$ & $5.945 \mathrm{E}-3$ & $3.123 \mathrm{E}-3$ & $1.423 \mathrm{E}-3$ & $4.896 \mathrm{E}-4$ \\
\hline $2^{-2}$ & $2.544 \mathrm{E}-2$ & $1.400 \mathrm{E}-2$ & $6.949 \mathrm{E}-3$ & $3.073 \mathrm{E}-3$ & $1.041 \mathrm{E}-3$ \\
\hline $2^{-4}$ & $7.254 \mathrm{E}-2$ & $4.364 \mathrm{E}-2$ & $2.489 \mathrm{E}-2$ & $1.248 \mathrm{E}-2$ & $4.380 \mathrm{E}-3$ \\
\hline $2^{-6}$ & $1.138 \mathrm{E}-1$ & $7.465 \mathrm{E}-2$ & $4.225 \mathrm{E}-2$ & $2.058 \mathrm{E}-2$ & $7.491 \mathrm{E}-3$ \\
\hline $2^{-8}$ & $1.284 \mathrm{E}-1$ & $8.256 \mathrm{E}-2$ & $4.944 \mathrm{E}-2$ & $2.812 \mathrm{E}-2$ & $1.121 \mathrm{E}-2$ \\
\hline $2^{-10}$ & $1.326 \mathrm{E}-1$ & $8.578 \mathrm{E}-2$ & $4.885 \mathrm{E}-2$ & $2.529 \mathrm{E}-2$ & $1.255 \mathrm{E}-2$ \\
\hline $2^{-12}$ & $1.337 \mathrm{E}-1$ & $8.687 \mathrm{E}-2$ & $4.958 \mathrm{E}-2$ & $2.460 \mathrm{E}-2$ & $9.732 \mathrm{E}-3$ \\
\hline $2^{-14}$ & $1.340 \mathrm{E}-1$ & $8.715 \mathrm{E}-2$ & $4.977 \mathrm{E}-2$ & $2.471 \mathrm{E}-2$ & $9.202 \mathrm{E}-3$ \\
\hline $2^{-16}$ & $1.341 \mathrm{E}-1$ & $8.722 \mathrm{E}-2$ & $4.981 \mathrm{E}-2$ & $2.474 \mathrm{E}-2$ & $9.213 \mathrm{E}-3$ \\
\hline $2^{-18}$ & $1.341 \mathrm{E}-1$ & $8.724 \mathrm{E}-2$ & $4.982 \mathrm{E}-2$ & $2.474 \mathrm{E}-2$ & $9.215 \mathrm{E}-3$ \\
\hline $2^{-20}$ & $1.341 \mathrm{E}-1$ & $8.724 \mathrm{E}-2$ & $4.983 \mathrm{E}-2$ & $2.475 \mathrm{E}-2$ & $9.216 \mathrm{E}-3$ \\
\hline$E_{N, \Delta t^{*}}$ & 0.13416 & 0.08724 & 0.04983 & 0.02812 & 0.01121 \\
\hline
\end{tabular}

Table 4

Numerical order of convergence for Example 2

\begin{tabular}{ccccc}
\hline$\varepsilon$ & $N=8$ & $N=16$ & $N=32$ & $N=64$ \\
\hline 1 & 0.766 & 0.929 & 1.134 & 1.539 \\
$2^{-2}$ & 0.861 & 1.011 & 0.996 & 1.561 \\
$2^{-4}$ & 0.733 & 0.810 & 1.038 & 1.511 \\
$2^{-6}$ & 0.609 & 0.821 & 0.814 & 1.458 \\
$2^{-8}$ & 0.637 & 0.740 & 0.950 & 1.326 \\
$2^{-10}$ & 0.629 & 0.812 & 1.011 & 1.010 \\
$2^{-12}$ & 0.623 & 0.809 & 1.010 & 1.338 \\
$2^{-14}$ & 0.621 & 0.808 & 1.010 & 1.425 \\
$2^{-16}$ & 0.621 & 0.808 & 1.010 & 1.425 \\
$2^{-18}$ & 0.621 & 0.808 & 1.010 & 1.425 \\
$2^{-20}$ & 0.621 & 0.808 & 0.814 & 1.010 \\
Min. & 0.609 & 0.740 & & \\
\hline
\end{tabular}

where

$$
f(x, y)=\sin (\pi x(1-x)) \sin (\pi y(1-y)) .
$$




\section{Appendix A}

In this appendix we expose the outlines to obtain the bounds for the solutions and their derivatives of problems (1.1) and (2.2), which are necessary for the uniform convergence analysis carried out in the previous sections.

Starting with problem (1.1) we will denote $D \equiv \Omega \times[0, T], S \equiv \partial \Omega \times[0, T]$, and we will suppose enough smoothness of functions $\vec{v}, k, f, u_{0}$ and also enough compatibility conditions (see [13, p. 206; 15 , pp. 319-320]) between initial and boundary data, in such way that for $l$ sufficiently large and a not integer $\alpha$, the solution of (1.1) satisfies

$$
u(x, y, t) \in C^{l+\alpha, l+\alpha,(l+\alpha) / 2}(\bar{\Omega} \times[0, T]) .
$$

Among these compatibility conditions we will remark the following one:

$$
f(0,0, t)=f(0,1, t)=f(1,0, t)=f(1,1, t)=0, \quad \forall t \in[0, T] .
$$

This condition is required for the asymptotic study which we carry out on the semidiscrete in time solutions. It was shown in [20,21] that the solution of problem (1.1) can be decomposed in two addenda $u=U+V$, where $U$ and $V$ are the regular and singular parts of $u$, respectively, which are defined as solutions of the following problems. On the one hand, $V$ is the solution of

$$
\begin{array}{ll}
\frac{\partial V}{\partial t}-\varepsilon \Delta V+\vec{v} \vec{\nabla} V+k V=0 & \text { in } D, \\
V(x, y, 0)=0 & \text { in } \Omega, \\
V(x, y, t)=-U(x, y, t) & \text { in } S .
\end{array}
$$

On the other hand, $U$ is the restriction to $D$ of the solution of

$$
\begin{array}{ll}
\frac{\partial U^{*}}{\partial t}-\varepsilon \Delta U^{*}+\vec{v}^{*} \vec{\nabla} U^{*}+k^{*} U^{*}=f^{*} & \text { in } D^{*} \equiv \Omega^{*} \times[0, T], \\
U^{*}(x, y, 0)=u_{0}^{*} & \text { in } \Omega^{*}, \\
U^{*}(x, y, t)=g^{*}(x, y, t) & \text { in } S^{*} \equiv \partial \Omega^{*} \times[0, T],
\end{array}
$$

where $\Omega^{*}$ is a smooth extension of $\Omega, \vec{v}^{*}, k^{*}, f^{*}$ are smooth extensions of $\vec{v}, k, f$ to $D^{*}, u_{0}^{*}$ is an extension of $u_{0}$ to $\Omega^{*}$ and $g^{*}$ is a smooth and compatible function. Since we have supposed $\vec{v}=\left(v_{1}, v_{2}\right)$ with $v_{i}(x, y) \geqslant \bar{v}>0, i=1,2$, the singular part $V$ can be decomposed in the form $V=V_{1}+V_{2}+\bar{V}_{1}$, where $V_{1}$ and $V_{2}$ are one-dimensional boundary layers in neighborhoods of sides $x=1$ and $y=1$ of $\Omega$, respectively, and $\bar{V}_{1}$ is a corner layer on neighborhood of $(1,1)$.

Function $V_{1}$ (and analogously $V_{2}$ ) can be obtained as a restriction to $D$ of the solution of

$$
\begin{array}{ll}
\frac{\partial V_{1}^{* *}}{\partial t}-\varepsilon \Delta V_{1}^{* *}+\vec{v}^{* *} \vec{\nabla} V_{1}^{* *}+k^{* *} V_{1}^{* *}=0 & \text { in } D^{* *} \equiv \Omega^{* *} \times[0, T], \\
V_{1}^{* *}(x, y, 0)=0 & \text { in } \Omega^{* *}, \\
V_{1}^{* *}(x, y, t)=-U^{* *} & \text { in } \partial \Omega_{1}^{* *} \times[0, T], \\
V_{1}^{* *}(x, y, t)=0 & \text { in } \partial \Omega_{2}^{* *} \times[0, T],
\end{array}
$$

where $\Omega^{* *}$ is a smooth extension of $\Omega$ near the vertex $(1,1)$ (see Fig. A.1), $\partial \Omega_{1}^{* *}$ is an extension of the boundary side $x=1$ beyond the point $(1,1)$ and $\partial \Omega_{2}^{* *}=\partial \Omega^{* *} \backslash \partial \Omega_{1}^{* *}$, and also $U^{* *}$ is a smooth 


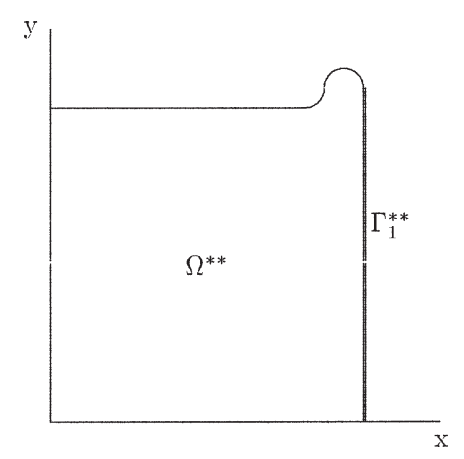

Fig. A.1.

and compatible extension of $U(1, y, t)$ to $\partial \Omega_{1}^{* *}$, as well as $\vec{v}^{* *}, k^{* *}$ are smooth extensions of $\vec{v}, k$ to $D^{* *}$.

The function $\bar{V}_{1}$ is the solution of

$$
\begin{array}{ll}
\frac{\partial \bar{V}_{1}}{\partial t}-\varepsilon \bar{V}_{1}+\vec{v} \vec{\nabla} \bar{V}_{1}+k \bar{V}_{1}=0 & \text { in } D, \\
\bar{V}_{1}(x, y, 0)=0 & \text { in } \Omega, \\
\bar{V}_{1}(x, y, t)=-\left(U+V_{1}+V_{2}\right) & \text { in } S .
\end{array}
$$

According to the results of [20,21], the following bounds are obtained:

$$
\begin{aligned}
& \left|\frac{\partial^{k_{s}+k_{t}} U(x, y, t)}{\partial x^{k_{x}} \partial y^{k_{y}} \partial t^{k_{t}}}\right| \leqslant C, \\
& \left|\frac{\partial^{k_{s}+k_{t}} V_{1}(x, y, t)}{\partial x^{k_{x}} \partial y^{k_{y}} \partial t^{k_{t}}}\right| \leqslant C \varepsilon^{-k_{x}} \exp \left(-\frac{\bar{v}(1-x)}{\varepsilon}\right), \\
& \left|\frac{\partial^{k_{s}+k_{t}} V_{2}(x, y, t)}{\partial x^{k_{x}} \partial y^{k_{y}} \partial t^{k_{t}}}\right| \leqslant C \varepsilon^{-k_{y}} \exp \left(-\frac{\bar{v}(1-y)}{\varepsilon}\right), \\
& \left|\frac{\partial^{k_{s}+k_{t}} \bar{V}_{1}(x, y, t)}{\partial x^{k_{x}} \partial y^{k_{y}} \partial t^{k_{t}}}\right| \leqslant C \varepsilon^{-k_{s}} \min \left\{\exp \left(-\frac{\bar{v}(1-x)}{\varepsilon}\right), \exp \left(-\frac{\bar{v}(1-y)}{\varepsilon}\right)\right\},
\end{aligned}
$$

where $k_{s}=k_{x}+k_{y}, k_{s}+2 k_{t} \leqslant l$ and $(x, y, t) \in \bar{\Omega} \times[0, T]$.

Now we study the behavior, with respect to the singular perturbation parameter $\varepsilon$, of the solutions of (2.2b) and their derivatives. An analogous development can be carried out for (2.2c). In fact, we will have to prove bounds ( $\varepsilon$-dependent) for $\widehat{u}^{n+1 / 2}(x, y)$ (respectively $\widehat{u}^{n+1}(x, y)$ ), solutions of $(2.2 \mathrm{~b}$ ) (respectively (2.2c)), and their $x$-derivatives (respectively $y$-derivatives) up to order 3 .

As a previous technical requirement, we will assume that

$$
\begin{array}{ll}
\left|L_{x, \varepsilon} u\left(x, y, t_{n}\right)\right| \leqslant C, \quad & \left|L_{x, \varepsilon}^{2} u\left(x, y, t_{n}\right)\right| \leqslant C, \\
\left|L_{y, \varepsilon} u\left(x, y, t_{n}\right)\right| \leqslant C, & \left|L_{y, \varepsilon}^{2} u\left(x, y, t_{n}\right)\right| \leqslant C .
\end{array}
$$


In the case $v_{1}=v_{1}(x), v_{2}=v_{2}(y), k_{1}=k_{1}(x), k_{2}=k_{2}(y)$, these assumptions can be immediately proved by the fact that functions $V \equiv L_{x, \varepsilon} u$ and $W \equiv L_{x, \varepsilon} V$ are the respective solutions of the initial-boundary value parabolic problems

$$
\begin{array}{ll}
\frac{\partial V}{\partial t}-\varepsilon \Delta V+\vec{v} \vec{\nabla} V+k V=L_{x, \varepsilon} f & \text { in } \Omega \times[0, T], \\
V(x, y, 0)=L_{x, \varepsilon} u_{0}(x, y) & \text { in } \Omega, \\
V(0, y, t)=f(0, y, t), V(1, y, t)=f(1, y, t) & \text { in }[0,1] \times[0, T], \\
V(x, 0, t)=V(x, 1, t)=0 & \text { in }[0,1] \times[0, T],
\end{array}
$$

and

$$
\begin{array}{ll}
\frac{\partial W}{\partial t}-\varepsilon \Delta W+\vec{v} \vec{\nabla} W+k W=L_{x, \varepsilon}^{2} f & \text { in } \Omega \times[0, T], \\
W(x, y, 0)=L_{x, \varepsilon} V(x, y, 0) & \text { in } \Omega, \\
W(0, y, t)=L_{x, \varepsilon} f(0, y, t)-\left(\frac{\partial f}{\partial t}(0, y, t)+L_{y, \varepsilon} f(0, y, t)\right) & \text { in }[0,1] \times[0, T], \\
W(1, y, t)=L_{x, \varepsilon} f(0, y, t)-\left(\frac{\partial f}{\partial t}(0, y, t)+L_{y, \varepsilon} f(1, y, t)\right) & \text { in }[0,1] \times[0, T], \\
W(x, 0, t)=W(x, 1, t)=0 & \text { in }[0,1] \times[0, T] .
\end{array}
$$

A maximum principle for these problems together with suitable smoothness and compatibility conditions for $u_{0}$ and $f$ give the desired bounds for $V$ and $W$. Analogously we can prove the same bounds for $L_{y, \varepsilon}, L_{y, \varepsilon}^{2}$.

For general $v_{1}(x, y), v_{2}(x, y), k_{1}(x, y)$ and $k_{2}(x, y)$, condition (A.2) is also true but not so easy to prove. In [21] it is shown that the corner layer $\bar{V}_{1}$ can be expanded in the form $\bar{V}_{1}=V^{0}+\varepsilon V^{1}+\bar{v}_{1}$, where $\bar{v}_{1}$ is the remainder term. Then, for $V_{1}, V_{2}$ and $\bar{v}_{1}$ it is easy to prove that $L_{x, \varepsilon} V_{1}, L_{x, \varepsilon}^{2} V_{1}, L_{x, \varepsilon} V_{2}$, $L_{x, \varepsilon}^{2} V_{2}, L_{x, \varepsilon} \bar{v}_{1}$ and $L_{x, \varepsilon}^{2} \bar{v}_{1}$ are bounded independently of $\varepsilon$ (similarly for $L_{y, \varepsilon}$ ). To obtain the same bounds for $V^{0}$ and $V^{1}$ we use analytical formulae, so we can deduce (A.2).

Note that $\left|\widehat{u}^{n+1 / 2}\right| \leqslant C$ by (2.1). In order to obtain bounds for its $x$-derivatives we will firstly take into account that

$$
\delta=\frac{\widehat{u}^{n+1 / 2}-u\left(t_{n}\right)}{\Delta t}
$$

is the solution of

$$
\begin{aligned}
& \left(I+\Delta t L_{x, \varepsilon}\right) \delta=-L_{x, \varepsilon} u\left(x, y, t_{n}\right)+f_{1}\left(x, y, t_{n+1}\right), \\
& \delta(0, y)=\delta(1, y)=0,
\end{aligned}
$$

where we have $\left|L_{x, \varepsilon} u\left(x, y, t_{n}\right)\right| \leqslant C$ in $\bar{\Omega}$ from the previous assumptions for the exact solution of the continuous problem. From (2.1), we obtain $|\delta| \leqslant C$.

Now, $\widehat{u}^{n+1 / 2}$ can be given as the solution to

$$
\begin{aligned}
& L_{x, \varepsilon} \widehat{u}^{n+1 / 2}=-\delta+f_{1}\left(x, y, t_{n+1}\right), \\
& \widehat{u}^{n+1 / 2}(0, y)=\widehat{u}^{n+1 / 2}(1, y)=0,
\end{aligned}
$$


and proceeding in a similar way as in [12] we prove that

$$
\begin{aligned}
& \left|\frac{\partial^{i} \widehat{u}^{n+1 / 2}}{\partial x^{i}}(0, y)\right| \leqslant C, \quad 0 \leqslant i \leqslant 1, \\
& \left|\frac{\partial^{i} \widehat{u}^{n+1 / 2}}{\partial x^{i}}(1, y)\right| \leqslant C \varepsilon^{-i}, \quad 0 \leqslant i \leqslant 2,
\end{aligned}
$$

and

$$
\left|\frac{\partial \widehat{u}^{n+1 / 2}}{\partial x}\right| \leqslant C\left[1+\varepsilon^{-1} \exp \left(-\frac{\bar{v}(1-x)}{\varepsilon}\right)\right] .
$$

To obtain bounds for higher order $x$-derivatives of $\widehat{u}^{n+1 / 2}$, we differentiate (A.4) with respect to $x$ and obtain

$$
L_{x, \varepsilon} s(x, y)=\frac{\partial u\left(x, y, t_{n}\right) / \partial x-\partial \widehat{u}^{n+1 / 2} / \partial x}{\Delta t}+\frac{\partial f_{1}}{\partial x}-\frac{\partial v_{1}}{\partial x} \frac{\partial \widehat{u}^{n+1 / 2}}{\partial x}-\frac{\partial k_{1}}{\partial x} \widehat{u}^{n+1 / 2},
$$

where $s(x, y)=\partial \widehat{u}^{n+1 / 2} / \partial x$. By the same reasoning as we followed for (A.5), we can obtain

$$
\left|\frac{\partial^{2} \widehat{u}^{n+1 / 2}}{\partial x^{2}}\right| \leqslant C\left[1+\varepsilon^{-2} \exp \left(-\frac{\bar{v}(1-x)}{\varepsilon}\right)\right] \text {. }
$$

To do this we simply need to prove that

$$
\left|\frac{\partial u\left(x, y, t_{n}\right) / \partial x-\partial \widehat{u}^{n+1 / 2} / \partial x}{\Delta t}\right| \leqslant C\left[1+\varepsilon^{-1} \exp \left(-\frac{\bar{v}(1-x)}{\varepsilon}\right)\right] .
$$

Let $\bar{\delta}=L_{x, \varepsilon} \delta$, which satisfies

$$
\begin{aligned}
& \left(I+\Delta t L_{x, \varepsilon}\right) \bar{\delta}=-L_{x, \varepsilon}^{2} u\left(x, y, t_{n}\right)+L_{x, \varepsilon} f_{1}\left(x, y, t_{n+1}\right), \\
& \bar{\delta}(0, y)=\frac{1}{\Delta t}\left(f_{1}\left(0, y, t_{n+1}\right)-L_{x, \varepsilon} u\left(0, y, t_{n}\right)\right) \\
& \bar{\delta}(1, y)=\frac{1}{\Delta t}\left(f_{1}\left(1, y, t_{n+1}\right)-L_{x, \varepsilon} u\left(1, y, t_{n}\right)\right) .
\end{aligned}
$$

The required compatibility conditions and the decomposition for $f$ given in (4.1), $\left|L_{x, \varepsilon}^{2} u\left(x, y, t_{n}\right)\right| \leqslant C$, $L_{x, \varepsilon} u\left(0, y, t_{n}\right)=f\left(0, y, t_{n}\right)=f_{1}\left(0, y, t_{n}\right)$ and $L_{x, \varepsilon} u\left(1, y, t_{n}\right)=f\left(1, y, t_{n}\right)=f_{1}\left(1, y, t_{n}\right)$. Therefore $|\bar{\delta}| \leqslant C$ in $\bar{\Omega}$. Then, in the problem

$$
L_{x, \varepsilon} \delta=\bar{\delta}, \quad \delta(0, y)=\delta(1, y)=0,
$$

we apply the Kellogg and Tsan technique [12] and it follows readily that

$$
\left|\frac{\partial \delta}{\partial x}\right| \leqslant C\left[1+\varepsilon^{-1} \exp \left(-\frac{\bar{v}(1-x)}{\varepsilon}\right)\right], \quad \forall(x, y) \in \bar{\Omega},
$$

and hence (A.7).

To obtain the corresponding bound for the third derivative we use a similar idea, differentiating (A.5), constructing a problem like (A.8) for $L_{x, \varepsilon}^{2} w$ and taking into account (4.1) and the compatibility conditions. 
Finally, the proof of (3.2)-(3.4) is finished in the following way. Let

$$
w_{1}(x, y)=\exp \left(-\frac{v_{1}(1, y)(1-x)}{\varepsilon}\right), \quad c_{y}=\frac{\varepsilon}{v_{1}(1, y)} \frac{\partial \widehat{u}^{n+1 / 2}}{\partial x}(1, y),
$$

and $z(x, y)=\widehat{u}^{n+1 / 2}-c_{y} w_{1}$. Then, it holds that

$$
|z(0, y)| \leqslant C, \quad|z(1, y)| \leqslant C, \quad\left|\frac{\partial z}{\partial x}(0, y)\right| \leqslant C, \quad \frac{\partial z}{\partial x}(1, y)=0,
$$

and

$$
L_{x, \varepsilon} z=-\delta+f_{1}-k_{1} \widehat{u}^{n+1 / 2}+c_{y}\left(v_{1}(1, y)-v_{1}(x, y)\right) \frac{\partial w_{1}}{\partial x}+k_{1} z \equiv g(x, y),
$$

being $g$ is a bounded function.

Differentiating (A.9) with respect to $x$ we obtain

$$
L_{x, \varepsilon} \frac{\partial z}{\partial x}=\frac{\partial g(x, y)}{\partial x}-\frac{\partial v_{1}}{\partial x} \frac{\partial z}{\partial x}-\frac{\partial k_{1}}{\partial x} z \equiv g_{1}(x, y) .
$$

Notice that

$$
\left|\frac{\partial \delta}{\partial x}\right| \leqslant C\left[1+\varepsilon^{-1} \exp \left(-\frac{\bar{v}(1-x)}{\varepsilon}\right)\right]
$$

implies

$$
\left|g_{1}(x, y)\right| \leqslant C\left[1+\varepsilon^{-1} \exp \left(-\frac{\bar{v}(1-x)}{\varepsilon}\right)\right] .
$$

Also the boundary conditions for $\partial z / \partial x$ are uniformly bounded. Then it follows that

$$
\left|\frac{\partial^{i} z}{\partial x^{i}}\right| \leqslant C\left[\left(1+\varepsilon^{1-i} \exp \left(-\frac{\bar{v}(1-x)}{\varepsilon}\right)\right], \quad 1 \leqslant i \leqslant 2 .\right.
$$

The proof of a bound similar to (A.11) for $i=3$ consists of differentiating (A.10) with respect to $x$, taking the corresponding boundary conditions which satisfy the bounds

$$
\left|\frac{\partial^{2} z}{\partial x^{i}}(0, y)\right| \leqslant C, \quad\left|\frac{\partial^{2} z}{\partial x^{i}}(1, y)\right| \leqslant C \varepsilon^{-1},
$$

and using again the same technique.

The bounds for the $y$-derivatives of $\widehat{u}^{n+1 / 2}$ are obtained by $y$-differentiating the differential equation in $(2.2 \mathrm{~b})$, considering the available bounds for the corresponding right hand side terms and also noticing the corresponding boundary conditions are null.

\section{Acknowledgements}

The authors thank Dr. F.J. Sayas and the referees for their helpful comments. 


\section{References}

[1] C. Clavero, J.C. Jorge and F. Lisbona, Splitting-time and exponential fitting space discretizations for diffusion-reaction problems, in: C. Perelló, C. Simó and J. Sola-Morales, eds., Proceedings International Conference on Differential Equations (World Scientific, Barcelona, 1991) 398-403.

[2] C. Clavero, J.C. Jorge and F. Lisbona, Uniformly convergent schemes for singular perturbation problems combining alternating directions and exponential fitting techniques, in: J.J.H. Miller, ed., Applications of Advanced Computational Methods for Boundary and Interior Layers (Boole Press, Dublin, 1993) 33-52.

[3] C. Clavero, J.C. Jorge, F. Lisbona and G.I. Shishkin, Splitting time methods and one dimensional special meshes for reaction-diffusion parabolic problems, in: Lecture Notes in Computer Science (Springer, Berlin, 1997) pp. 106-113.

[4] C. Clavero, J.J.H. Miller, E. O'Riordan and G.I. Shishkin, An accurate numerical solution of a two dimensional heat transfer problem with a parabolic boundary layer, J. Comput. Math. 16 (1998) 27-39.

[5] P.A. Farrell, Sufficient conditions for uniform convergence of a class of difference schemes for a singularly perturbed problem, IMA J. Numer. Anal. 7 (1987) 459-472.

[6] E.C. Gartland, Uniform high-order difference schemes for a singularly perturbed two point boundary value problem, Math. Comp. 48 (1987) 551-564.

[7] E.C. Gartland, Graded-mesh difference schemes for singularly perturbed two-point boundary value problems, Math. Comp. 51 (1988) 631-657.

[8] A.F. Hegarty, J.J.H. Miller, E. O'Riordan and G.I. Shishkin, Special numerical methods for convectiondominated laminar flows at arbitrary Reynolds number, East-West J. Numer. Math. 2 (1994) 65-74.

[9] A.F. Hegarty, J.J.H. Miller, E. O'Riordan and G.I. Shishkin, Special meshes for finite difference approximations to an advection-diffusion equation with parabolic layers, J. Comput. Phys. 117 (1995) $47-54$.

[10] D. Herceg, Uniform fourth order difference scheme for a singular perturbation problem, Numer. Math. 56 (1990) 675-693.

[11] J.C. Jorge and F. Lisbona, Contractivity results for alternating direction schemes in Hilbert spaces, Appl. Numer. Math. 15 (1994) 65-75.

[12] R.B. Kellogg and A. Tsan, Analysis of some difference approximations for a singular perturbation problem without turning points, Math. Comp. 32 (1978) 1025-1039.

[13] H.O. Kreiss and J. Lorenz, Initial-Boundary Value Problems and the Navier-Stokes Equations (Academic Press, New York, 1989).

[14] R.B. Kellogg and S. Shih, Asymptotic analysis of a singular perturbation problem, SIAM J. Math. Anal. 18 (1987) 1467-1511.

[15] O.A. Ladyzenskaja, V.A. Solonikov and N.N. Ural'ceva, Linear and quasilinear equations of parabolic type, J. ACM 23 (1968).

[16] J.J.H. Miller, E. O'Riordan and G.I. Shishkin, Fitted Numerical Methods for Singular Perturbation Problems. Error Estimates in the Maximum Norm for Linear Problems in One and Two Dimensions (World Scientific, 1996).

[17] E. O'Riordan and M. Stynes, A globally convergent finite element method for a singularly perturbed elliptic problem in two dimensions, Math. Comp. 57 (1991) 47-62.

[18] H.G. Roos, M. Stynes and L. Tobiska, Numerical Methods for Singularly Perturbed Differential Equations (Springer, Berlin, 1996).

[19] G.I. Shishkin, Approximation of the solution to singularly perturbed boundary value problems with boundary layers, Comput. Math. Math. Phys. 29 (1989) 1-10.

[20] G.I. Shishkin, Grid approximation of singularly perturbed boundary value problems with convective terms, Soviet. J. Numer. Anal. Math. Modeling 5 (1990) 173-187. 
[21] G.I. Shishkin, Grid Approximations of Singularly Perturbed Elliptic and Parabolic Equations (Russian Academy of Sciences, Ural Branch, Ekaterinburg, 1992) (in Russian).

[22] G. Sun and M. Stynes, Finite element methods for singularly perturbed higher order elliptic two point boundary value problems I: reaction-diffusion type problems, IMA J. Numer. Anal. 15 (1995) 117-139.

[23] G. Sun and M. Stynes, Finite element methods for singularly perturbed higher order elliptic two point boundary value problems II: convection-diffusion type problems, IMA J. Numer. Anal. 15 (1995) 197-219.

[24] M. Stynes and H.G. Roos, The midpoint upwind scheme, Appl. Numer. Math. 23 (1997) 361-374.

[25] N.N. Yanenko, The Method of Fractional Steps (Springer, Berlin, 1971). 